\title{
Comparison of Hematological Parameters and Critical Value Analysis in the Prevention and Control of Thalassaemia in Hainan Li and Han Nationalities
}

\author{
Junjie Hu, Xinping Chen, Xiaojuan Li, Weihua Xu, Shengmiao Fu \\ Department of Central Laboratory, Hainan General Hospital/Hainan Affiliated Hospital of Hainan Medical University, Hainan \\ Provincial Key Laboratory for Cell and Molecular Genetic Translational Medicine, Haikou, China \\ Email: chenxinping52@126.com,smfu2000@126.com
}

How to cite this paper: Hu, J.J., Chen, X.P., Li, X.J., Xu, W.H. and Fu, S.M. (2021) Comparison of Hematological Parameters and Critical Value Analysis in the Prevention and Control of Thalassaemia in Hainan $\mathrm{Li}$ and Han Nationalities. Open Journal of Blood Diseases, 11, 46-56.

https://doi.org/10.4236/ojbd.2021.112006

Received: April 13, 2021

Accepted: May 25, 2021

Published: May 28, 2021

Copyright $\odot 2021$ by author(s) and Scientific Research Publishing Inc. This work is licensed under the Creative Commons Attribution International License (CC BY 4.0).

http://creativecommons.org/licenses/by/4.0/

\begin{abstract}
Objective: To compare the distribution of "mean corpuscular hemoglobin"-MCV, "mean corpuscular volume"-MCH, "hemoglobin"-HGB, "hemoglobin $\mathrm{A}$ "- $\mathrm{HbA}$ and "hemoglobin $\mathrm{A} 2$ "- $\mathrm{HbA} 2$ in $\alpha$ and $\beta$ thalassemia hematology screening between Li and Han nationality, and analyze the best diagnostic cut-off value. Methods: Select 7816 middle school students from Li nationality area as the research object, collect peripheral blood for blood cell analysis, hemoglobin electrophoresis and thalassaemia gene detection, and compare the difference in hematological parameters of common thalassemia genotype between $\mathrm{Li}$ and $\mathrm{Han}$ nationalities. Taking the genetic test results as the gold standard, construct the receiver operator characteristic curve (ROC curve) of relevant hematology parameters, calculate the Youden index and take its maximum diagnostic cut-off point as the best critical value. Results: Comparison of hematological parameters of common thalassemia genotypes showed that the average value of MCH and MCV of - $\alpha 3.7 /-a 4.2$ type in Li nationality was lower than that of Han nationality, and the average value of $\mathrm{HbA} 2$ of $\mathrm{CD} 41-42 / \beta \mathrm{N}$ type was higher than that of Han nationality, there was no significant difference among other genotypes. ROC curve analysis shows that the MCH, MCV, and HGB values have poor diagnostic efficiency for thalassaemia, HbA has a slightly better diagnostic efficiency for $\alpha$ thalassaemia, and the optimal cut-off values of $\mathrm{HbA}$ for $\mathrm{Li}$ and $\mathrm{Han}$ nationalities are 96.95\% and $97.75 \%$, respectively; $\mathrm{HbA} 2$ has better screening efficiency for $\beta$-thalassemia, and the optimal cut-off values of $\mathrm{HbA} 2$ for $\mathrm{Li}$ and Han nationalities are $4.20 \%$ and $3.45 \%$ respectively. Conclusion: In the prevention and control screening of thalassaemia in the Li and Han nationalities, hemoglobin electrophoresis technology has a better diagnostic efficiency.
\end{abstract}




\section{Keywords}

Blood Cell Analysis, Hemoglobin Electrophoresis, Gene Detection, ROC Curve

\section{Introduction}

Thalassemia also called globinogenesis anemia. According to epidemiological survey data, the carrying rate of $\alpha$-thalassaemia among the Li ethnic group and the Han ethnic group is $52.19 \%$ and $19.11 \%$ respectively, and the carrying rate of $\beta$-thalassaemia among the $\mathrm{Li}$ ethnic group and the Han ethnic group is $1.86 \%$ and $1.95 \%$ respectively [1]. The current epidemic form of thalassemia in Hainan is very severe. Although genetic testing technology can achieve accurate detection of thalassaemia, due to practical problems such as manpower, material resources, medical and health conditions, and ethnic regional differences, largescale genetic testing for thalassaemia is temporarily impossible in Hainan. Hematology screening is performed first, and if the initial screening result is positive, then the thalassaemia gene test is carried out. This is a screening mode generally adopted by relevant government departments, research teams and primary medical institutions for the prevention and control of thalassaemia. The hematology screening index for thalassaemia in Hainan has not yet been established. Generally, the index commonly used internationally or used in Guangdong and Guangxi is used as a reference. However, the parameter values of hematology screening will be affected by factors such as different regions, different nationalities, and differences in testing instruments. Combining the previous research foundation and new survey data, this study intends to compare the results of blood cell analysis, hemoglobin electrophoresis and thalassaemia gene detection between $\mathrm{Li}$ and Han people. Count the distribution ranges of MCV, MCH, HGB, $\mathrm{HbA}, \mathrm{HbA} 2$ values in common $\alpha$ and $\beta$ thalassaemia genotypes, and use the results of genetic testing as the gold standard to analyze the best cut-off values. This study collected thalassaemia detection data in most Li nationality areas in Hainan, covering a wide range and reliable data sources. The research results can provide a more accurate reference for $\mathrm{Li}$ and Han thalassaemia screening. Increase the feasibility of government departments and primary medical institutions to implement thalassemia prevention and control screening.

\section{Object and Method}

\subsection{Object}

From June 2015 to June 2018, in Qiongzhong, Baisha, Changjiang, Lingshui, Wuzhishan, Baoting and other Li ethnic areas, 7816 middle school students (14 18 years old) were randomly selected as the research objects. There are 5259 people of Li nationality and 2557 people of Han nationality. Selection and exclusion criteria: Li nationality candidates are required to have their parents both $\mathrm{Li}$ 
nationality, and Han nationality candidates are required that their parents are Han nationality. If one parent is of another ethnicity, they will be excluded. At the same time, the study subject has a family history of hemophilia and other blood-borne diseases cannot join the group. Subjects and guardians obtained 2 $\mathrm{mL}$ of peripheral venous blood after informed consent, EDTA anticoagulation, and stored and transported at $4^{\circ} \mathrm{C}$.

\subsection{Method}

\subsubsection{Blood Cell Analysis}

The XE-2100 five-differentiation hematology analyzer produced by Japanese sysmex company is used for blood cell analysis. All blood samples are required to be tested within 6 hours.

\subsubsection{Capillary Electrophoresis}

Hemoglobin electrophoresis analysis is performed with Cappilarys 2 automatic capillary electrophoresis analyzer produced by Sebia, France. The samples must be stored at $-80^{\circ} \mathrm{C}$ and the test should be completed within 2 weeks.

\subsubsection{Genetic Testing}

Using the Luminex MAGPIX scanner produced by R\&D Systems in the United States and the Thalassemia $(\alpha / \beta)$ gene detection kit produced by Guangzhou Sun Yat-sen University Daan Gene Co., Ltd. to detect the three deletion types related to $\alpha$-thalassemia (-SEA, $-\alpha 3.7$ and $-\alpha 4.2$ ) and 3 mutants (WS122, QS125 and CS142) and 17 mutants related to $\beta$-thalassaemia (IVS-1-5, -29, CD31, Cap, IVS-2-654, CD26, -32, CD71-72, IVS-1-1, CD41-42, CD17, -28, CD43, Int, CD14-15, CD27-28, -30).

\subsubsection{Statistical Analysis}

Using SPSS19.0 statistical software, the statistical methods were $\mathrm{x} 2$ test and $\mathrm{t}$ test for comparison of differences between groups, and $P<0.05$ was considered statistically significant. Using genetic testing as the gold standard, compare the hematological parameters related to $\alpha$ and $\beta$ thalassaemia of Li and Han nationalities with their normal control group respectively. Draw the ROC curve, calculate the Youden index (Yorden index $=$ sensitivity + specificity -1$)$, and use the maximum diagnostic cut-off point of the Youden index as the best cut-off value.

\section{Result}

\subsection{Genotype Distribution}

As shown in Table 1, the $\alpha$-thalassaemia genotype carrying rate of the Li ethnic group was $60.83 \%$ (3199/5259), and $17 \alpha$-thalassaemia genotypes were detected. Among them, the genotypes with a higher carrying rate were $-\alpha 3.7 / \alpha \alpha(15.59 \%)$, $-\alpha 4.2 / \alpha \alpha$ (14.34\%), $-\alpha \mathrm{WS} / \alpha \alpha$ (8.10\%), - $\alpha 3.7 /-\alpha 4.2$ (6.39\%), - $\alpha 3.7 /-\alpha 3.7$ (4.05\%), $-\alpha 4.2 /-\alpha \mathrm{WS}$ (3.08\%), $-\alpha 3.7 /-\alpha \mathrm{WS}$ (3.02\%), SEA/ $\alpha \alpha$ (1.54\%). In contrast, the $\alpha$-thalassaemia carrying rate of the Han ethnic group was $23.90 \%(611 / 2557)$, and $14 \alpha$-thalassaemia genotypes were detected, among which the genotype with 
Table 1. Distribution of $\alpha$ and $\beta$ thalassaemia genotypes.

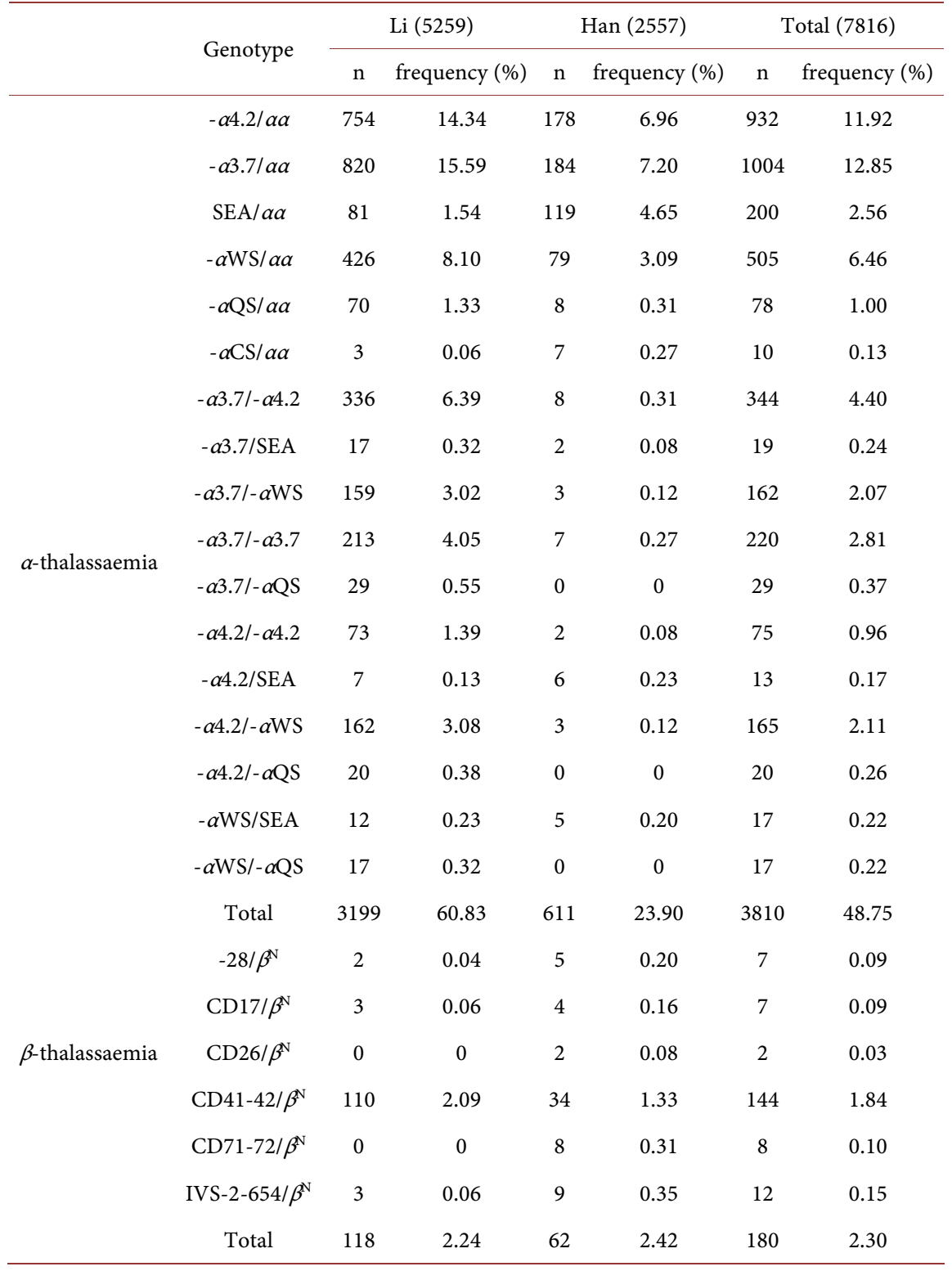

the higher carrying rate was $-\alpha 3.7 / \alpha \alpha(7.20 \%),-A 4.2 / \alpha \alpha(6.96 \%), \mathrm{SEA} / \alpha \alpha$ (4.65\%), $-\alpha \mathrm{WS} / \alpha \alpha$ (3.09\%), $-\alpha \mathrm{QS} / \alpha \alpha(0.31 \%),-\alpha 3.7 /-\alpha 4.2$ (0.31\%), $-\alpha \mathrm{CS} / \alpha \alpha$ (0.27\%), - $\alpha 3.7 /-\alpha 3.7$ (0.27\%). In the distribution of $\beta$-thalassaemia genotypes, 4 $\beta$-thalassaemia genotypes were detected in the Li nationality, and the carrying rate was $2.24 \%(118 / 5259)$. Six $\beta$-thalassemia genotypes were detected in the Han nationality, with a carrier rate of $2.42 \%(62 / 2557)$. Among them, the Li nationality and Han nationality have the highest $\mathrm{CD} 41-42 / \beta \mathrm{N}$ carrier rate, which are $2.09 \%$ and $1.33 \%$, respectively.

\subsection{Distribution and Comparison of Hematological Parameters Related to Common $\alpha$ and $\beta$ Thalassaemia Genotypes}

The distribution of hematology screening results (mean \pm standard deviation) is 
shown in Table 2 and Table 3. Statistics of related parameters of blood cell analysis showed that the average values of $\mathrm{MCH}, \mathrm{MCV}$, and $\mathrm{HGB}$ carrying $\mathrm{CD} 41-42 / \beta \mathrm{N}$ in $\mathrm{Li}$ and Han nationalities were lower than other common $\alpha$-thalassemia genotypes. In the $\mathrm{Li}$, the average values of $\mathrm{MCH}, \mathrm{MCV}$ and $\mathrm{HGB}$ of single mutation $(-\alpha \mathrm{WS} / \alpha \alpha)$, single deletion $(-\alpha 4.2 / \alpha \alpha,-\alpha 3.7 / \alpha \alpha)$ and single deletion combined with single mutation (- $\alpha 3.7 /-\alpha \mathrm{WS},-\alpha 4.2 /-\alpha \mathrm{WS})$ are higher than double deletion $(-\alpha 3.7 /-\alpha 3.7,-\alpha 3.7 /-\alpha 4.2, \mathrm{SEA} / \alpha \alpha)$. In the Han, the average values of $\mathrm{MCH}, \mathrm{MCV}$ and $\mathrm{HGB}$ of single mutant $(-\alpha \mathrm{WS} / \alpha \alpha,-\alpha \mathrm{QS} / \alpha \alpha,-\alpha \mathrm{CS} / \alpha \alpha)$ and single deletion $(-\alpha 4.2 / \alpha \alpha,-\alpha 3.7 / \alpha \alpha)$ are also higher than those of double deletion $(-\alpha 3.7 /-\alpha 3.7,-\alpha 3.7 /-\alpha 4.2, \mathrm{SEA} / \alpha \alpha)$. This means that the $\mathrm{MCH}, \mathrm{MCV}$, and HGB values of the Li and Han nationalities in $\alpha$-thalassaemia showed a downward trend as the degree of gene deletion increased $(P<0.05)$. Statistics of hemoglobin electrophoresis related parameters showed that the average $\mathrm{HbA} 2$ of

Table 2. The distribution of hematological parameter values of the common thalassemia genotypes in the Li nationality (mean \pm standard deviation).

\begin{tabular}{cccccc}
\hline Genotype & MCH $(\mathrm{pg})$ & MCV $(\mathrm{fL})$ & HGB $(\mathrm{g} / \mathrm{L})$ & HbA $(\%)$ & HbA2 $(\%)$ \\
\hline$-\alpha \mathrm{WS} / \alpha \alpha$ & $26.78 \pm 3.06$ & $82.41 \pm 5.74$ & $136.49 \pm 13.31$ & $96.57 \pm 1.41$ & $2.40 \pm 0.40$ \\
$-\alpha 4.2 / \alpha \alpha$ & $25.88 \pm 2.45$ & $80.79 \pm 5.86$ & $133.80 \pm 14.63$ & $96.90 \pm 1.47$ & $2.36 \pm 0.37$ \\
$-\alpha 3.7 / \alpha \alpha$ & $26.12 \pm 2.12$ & $81.45 \pm 5.13$ & $133.85 \pm 13.30$ & $96.91 \pm 1.35$ & $2.26 \pm 0.35$ \\
$-\alpha 3.7 /-\alpha \mathrm{WS}$ & $25.01 \pm 2.19$ & $78.35 \pm 5.75$ & $132.97 \pm 13.11$ & $96.68 \pm 1.33$ & $2.46 \pm 0.58$ \\
$-\alpha 4.2 /-\alpha \mathrm{WS}$ & $24.79 \pm 2.04$ & $77.94 \pm 5.39$ & $131.93 \pm 14.66$ & $96.74 \pm 1.35$ & $2.37 \pm 0.37$ \\
$-\alpha 3.7 /-\alpha 3.7$ & $22.56 \pm 1.84$ & $71.36 \pm 4.20$ & $127.10 \pm 12.10$ & $97.15 \pm 1.25$ & $2.15 \pm 0.25$ \\
$-\alpha 3.7 /-\alpha 4.2$ & $22.62 \pm 2.17$ & $72.06 \pm 5.26$ & $129.43 \pm 12.48$ & $97.07 \pm 1.45$ & $2.18 \pm 0.40$ \\
SEA/ $\alpha \alpha$ & $21.50 \pm 2.44$ & $69.32 \pm 6.67$ & $124.30 \pm 11.68$ & $96.71 \pm 1.99$ & $2.04 \pm 0.30$ \\
Normal & $25.32 \pm 3.52$ & $79.03 \pm 8.83$ & $132.11 \pm 20.47$ & $96.51 \pm 2.08$ & $2.54 \pm 0.92$ \\
CD41-42/ $\beta^{\mathrm{N}}$ & $19.69 \pm 2.07$ & $63.25 \pm 5.05$ & $117.08 \pm 14.45$ & $93.88 \pm 1.45$ & $5.11 \pm 0.55$ \\
\hline
\end{tabular}

Table 3. Distribution of hematological parameter values of common thalassaemia genotypes in the Han nationality (mean \pm standard deviation).

\begin{tabular}{cccccc}
\hline Genotype & MCH (pg) & MCV (fL) & HGB (g/L) & HbA (\%) & HbA2 (\%) \\
\hline$-\alpha \mathrm{WS} / \alpha \alpha$ & $26.79 \pm 1.88$ & $84.42 \pm 4.46$ & $135.40 \pm 12.57$ & $96.63 \pm 1.58$ & $2.32 \pm 0.32$ \\
$-\alpha \mathrm{QS} / \alpha \alpha$ & $27.11 \pm 2.65$ & $83.87 \pm 8.13$ & $134.78 \pm 10.39$ & $96.91 \pm 1.69$ & $2.44 \pm 0.96$ \\
$-\alpha \mathrm{CS} / \alpha \alpha$ & $26.85 \pm 1.89$ & $81.28 \pm 3.50$ & $135.33 \pm 13.24$ & $97.04 \pm 0.86$ & $2.38 \pm 0.20$ \\
$-\alpha 4.2 / \alpha \alpha$ & $26.33 \pm 1.97$ & $81.83 \pm 5.05$ & $133.96 \pm 16.83$ & $97.00 \pm 2.38$ & $2.31 \pm 0.32$ \\
$-\alpha 3.7 / \alpha \alpha$ & $26.66 \pm 2.16$ & $82.66 \pm 5.48$ & $134.66 \pm 13.97$ & $96.83 \pm 2.10$ & $2.25 \pm 0.33$ \\
$-\alpha 3.7 /-\alpha 3.7$ & $24.21 \pm 3.50$ & $75.80 \pm 6.16$ & $130.10 \pm 8.31$ & $97.53 \pm 0.61$ & $2.20 \pm 0.26$ \\
$-\alpha 3.7 /-\alpha 4.2$ & $24.66 \pm 4.30$ & $76.27 \pm 10.24$ & $125.50 \pm 11.66$ & $96.06 \pm 2.39$ & $2.15 \pm 0.24$ \\
SEA/ $\alpha \alpha$ & $21.83 \pm 2.85$ & $68.98 \pm 7.07$ & $124.61 \pm 12.55$ & $96.92 \pm 3.31$ & $2.06 \pm 0.25$ \\
Normal & $27.98 \pm 10.27$ & $85.00 \pm 8.15$ & $136.90 \pm 16.99$ & $96.69 \pm 2.55$ & $2.48 \pm 0.61$ \\
CD41-42/ $\beta^{N}$ & $19.74 \pm 2.42$ & $63.61 \pm 6.58$ & $121.09 \pm 17.12$ & $93.98 \pm 1.73$ & $4.92 \pm 0.79$ \\
\hline
\end{tabular}


$\mathrm{CD} 41-42 / \beta \mathrm{N}$ in $\mathrm{Li}$ and Han nationalities was significantly higher than other common $\alpha$-thalassemia genotypes, while the $\mathrm{HbA}$ value was significantly lower than $\alpha$-thalassemia $(P<0.05)$. Statistics of hemoglobin electrophoresis related parameters showed that the average HbA2 of CD41-42/ $\beta \mathrm{N}$ in Li and Han nationalities was significantly higher than other common $\alpha$-thalassemia genotypes, while the $\mathrm{HbA}$ value was significantly lower than $\alpha$-thalassemia $(P<0.05)$. In $\alpha$-thalassaemia, there was no significant difference in the average $\mathrm{HbA}$ between the Li and Han genotypes $(P>0.05)$, while the double deletion type $(-\alpha 3.7 /-\alpha 3.7$, $-\alpha 3.7 /-\alpha 4$. 2. The average HbA2 of SEA/ $\alpha \alpha)$ was lower than that of other genotypes $(P<0.05)$.

The comparison of the same thalassaemia genotypes of different ethnic groups showed that the mean values of $\mathrm{MCH}$ and $\mathrm{MCV}$ of the $\alpha 3.7 /$ - $\alpha 4.2$ genotype of the Li nationality were lower than those of the Han nationality $(P<0.05)$, and there was no significant difference among other genotypes (Figure 1(A) and Figure 1(B)). For HGB and HbA, there was no significant difference between the genotypes of $\mathrm{Li}$ and Han nationalities (Figure 1(C) and Figure 1(D), $P>$ 0.05). Another important result is that the average $\mathrm{HbA} 2$ of $\mathrm{CD} 41-42 / \beta \mathrm{N}$ genotype of Li nationality is higher than that of Han nationality $(\mathrm{P}<0.05)$, and there is no significant difference among other genotypes (Figure 1(E)).

\subsection{Analysis of Critical Value of Relevant Parameters in Hematology Screening}

Use SPSS software to draw the ROC curve of $\mathrm{MCH}, \mathrm{MCV}, \mathrm{HGB}, \mathrm{HbA}$ and HbA2 for screening $\alpha$ and $\beta$ thalassaemia, calculate the Youden index and conduct critical value analysis. In a-thalassaemia screening, the Li nationality has the highest screening value for $\mathrm{HbA}$, the area under the curve (AUC) is 0.574 , maximum Youden index is 0.139 , optimal threshold for $\mathrm{HbA}$ is $96.95 \%$, sensitivity and specificity are 0.653 and 0.486 respectively (Figure 2(A)). For the Han nationality, the highest screening value is $\mathrm{HbA}$, with an AUC of 0.543 and a maximum Youden index of 0.090 , corresponding to the best cut-off value of $\mathrm{HbA}$ of $97.75 \%$, with sensitivity and specificity of 0.306 and 0.784 , respectively (Figure $2(\mathrm{C})$ ). In $\beta$-thalassaemia screening, the Li nationality has the highest screening value for $\mathrm{HbA} 2$, AUC is 0.975 , and the maximum Youden index is 0.943 , corresponding to the optimal cutoff value of $\mathrm{HbA} 2$ at $4.20 \%$, and the sensitivity and specificity are 0.953 and 0.990 , respectively (Figure 2(B)); Similarly, the Han nationality has the highest screening value for $\mathrm{HbA2}$, with an AUC of 0.974 and a maximum Youden index of 0.936 , corresponding to the optimal cutoff value of $\mathrm{HbA} 2$ of $3.45 \%$, with sensitivity and specificity of 0.938 and 0.998 respectively (Figure 2(D)).

\section{Discussion}

Blood cell analysis and hemoglobin electrophoresis have always been common methods for screening for thalassaemia. According to the detection values of 

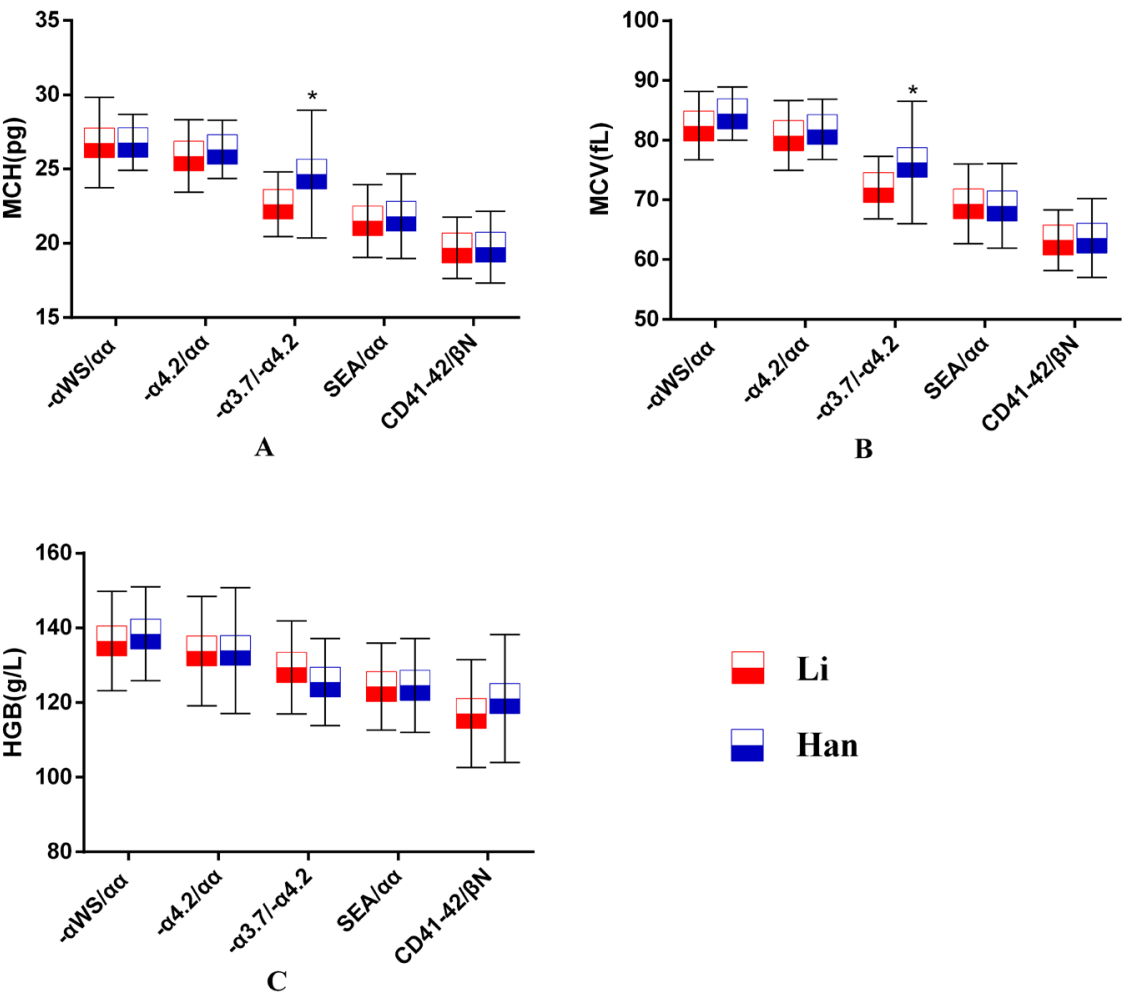

Li

Han
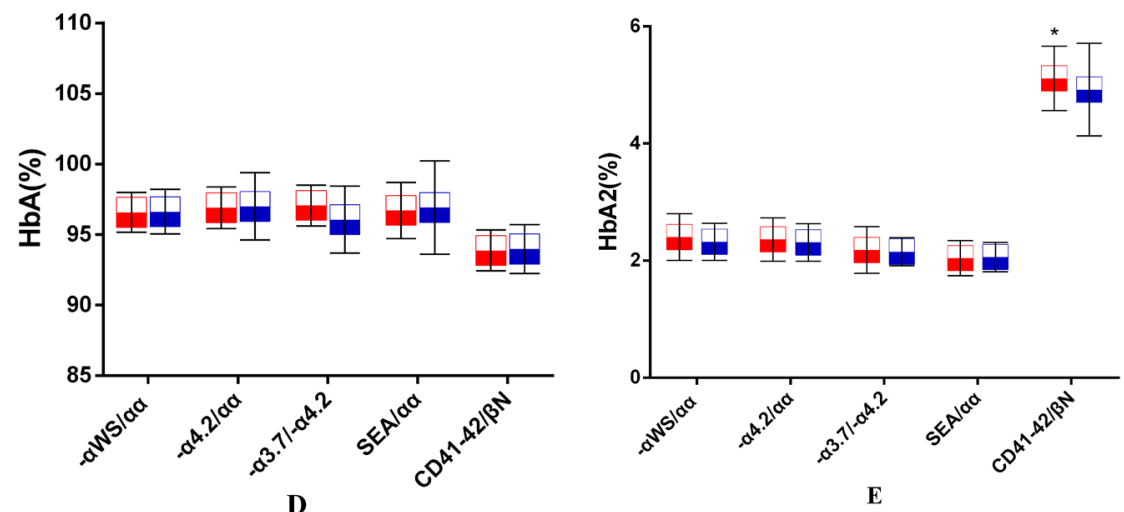

Figure 1. Comparison of hematology parameters between Li nationality (Li) and Han nationality (Han) (mean \pm standard deviation).

$\mathrm{MCV}, \mathrm{MCH}, \mathrm{HGB}$ in blood cell analysis or $\mathrm{HbA}, \mathrm{HbA} 2$ in hemoglobin electrophoresis, the preliminary screening of thalassaemia is carried out [2] [3] [4] [5]. Commonly used indicators are suspected carriers of thalassaemia with MCV < $82 \mathrm{fl}$ or $\mathrm{MCH}<27 \mathrm{pg}$, or $\mathrm{HbA}: 96.5 \%$ - 97.5\%, HbA2: $2.4 \%-3.0 \%$, HbF: $0 \%-$ $1.0 \%$ to exclude thalassaemia carriers. Although it can increase the detection rate of thalassaemia to a certain extent, its sensitivity and specificity often vary depending on the region and the tested nationality [6]. Foreign researchers suggest that multiple hematological parameters should be combined for testing in thalassaemia screening to improve accuracy and reduce misdiagnosis [7] [8]. In the previous work, we used the above indicators to evaluate the role of blood cell 


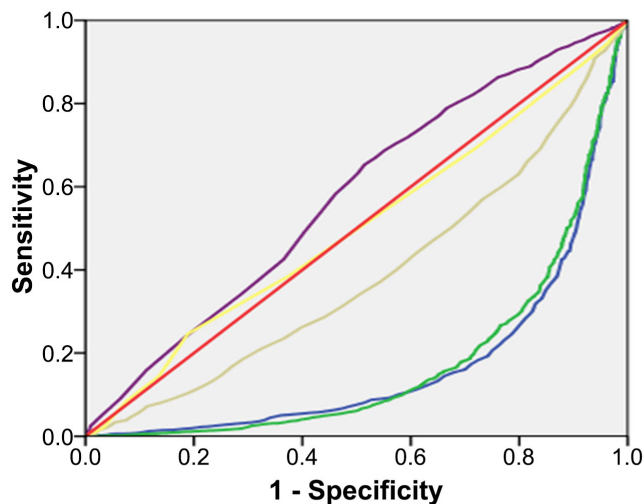

A

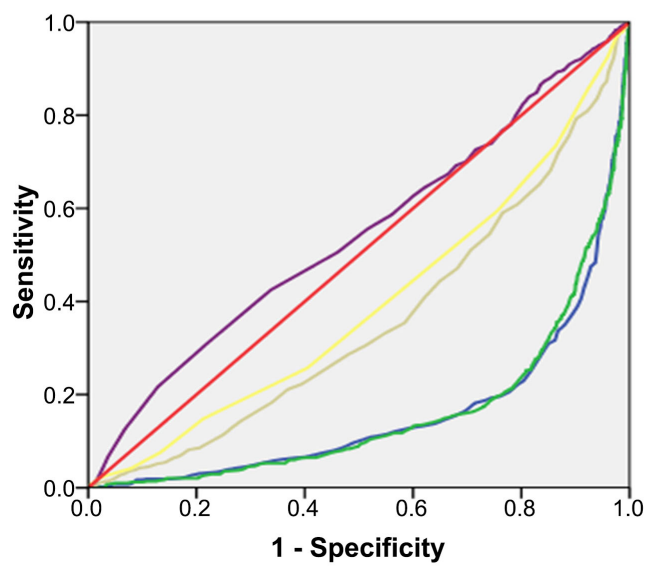

C

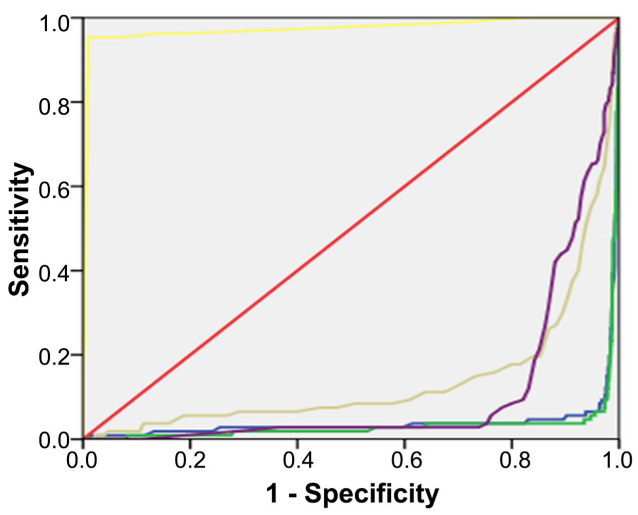

B

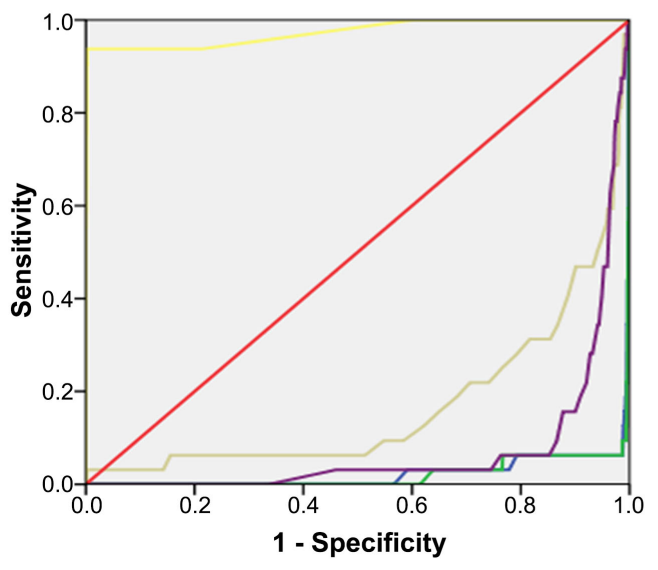

D

Figure 2. ROC curve of hematological parameter values for thalassaemia screening in Li and Han nationalitie.

analysis and hemoglobin electrophoresis in the screening of thalassaemia in the $\mathrm{Li}$ nationality. The results showed that the sensitivity of $\mathrm{Li}$ and Han blood cell analysis to diagnose thalassaemia was $76.59 \%$ and $70.19 \%$, and the specificity was $74.54 \%$ and $71.82 \%$, respectively; The sensitivity of hemoglobin electrophoresis to diagnose thalassaemia was $79.05 \%$ and $93.27 \%$, and the specificity was $63.31 \%$ and $54.01 \%$, respectively; The sensitivity of the combined detection and diagnosis of thalassaemia by the two methods was $99.41 \%$ and $98.40 \%$, and the specificity was $46.75 \%$ and $34.32 \%$, respectively [9].

The reference values of hematology screening obtained by different clinical laboratories and research teams are different. For example, Lin Jinduan et al. [10] analyzed the thalassaemia of early-pregnant women in Guangdong and found that the best cutoff values for MCV and MCH for thalassaemia were 79.7 $\mathrm{fL}$ and $26.7 \mathrm{pg}$, respectively. He Tianwen et al. [11] analyzed and found that the optimal cut-off values for HbA2 value to screen stationary, mild, intermediate $\alpha$-thalassaemia and mild $\beta$-thalassaemia were $2.85 \%, 2.65 \%$ and $2.25 \%$, and 3 . $45 \%$, respectively. This study carried out thalassaemia genetic testing on the Li and Han nationalities in Hainan and found that the carrying rates of $\alpha$-thalassaemia and $\beta$-thalassaemia were $60.83 \%$ and $2.24 \%$ for the Li nationality in Hai- 
nan, and $23.90 \%$ and $2.42 \%$ for the Han nationality. The results show that the prevalence of thalassaemia in the two ethnic groups is dominated by $\alpha$-thalassaemia, and the carrying rate of $\alpha$-thalassaemia and $\beta$-thalassaemia is quite different. In $\alpha$-thalassaemia, the average values of $\mathrm{MCH}, \mathrm{MCV}$, and $\mathrm{HGB}$ of related genotypes of $\mathrm{Li}(-\alpha \mathrm{WS} / \alpha \alpha,-\alpha 4.2 / \alpha \alpha,-\alpha 3.7 / \alpha \alpha,-\alpha 3.7 /-\alpha \mathrm{WS},-\alpha 4.2 /-\alpha \mathrm{WS})$ and Han $(-\alpha \mathrm{WS} / \alpha \alpha,-\alpha \mathrm{QS} / \alpha \alpha,-\alpha \mathrm{CS} / \alpha \alpha,-\alpha 4.2 / \alpha \alpha,-\alpha 3.7 / \alpha \alpha)$ nationalities were compared with their respective normal groups, the results showed no significant difference. Therefore, it is speculated that the diagnostic value of $\mathrm{MCH}, \mathrm{MCV}$, and $\mathrm{HGB}$ for thalassaemia may be poor. In $\beta$-thalassaemia, there are fewer genotypes detected, and they are mainly carrying CD $41-42 / \beta \mathrm{N}$. Statistical analysis shows that the average $\mathrm{HbA} 2$ is significantly higher than the common genotypes of $\alpha$-thalassaemia and the normal control group. The value of HbA2 in the Li and Han nationalities may have better diagnostic value for $\beta$-thalassemia.

The ROC curve can be used to compare two or more experimental results to the diagnosis ability of a certain disease. Draw the ROC curve of each experimental result to the same coordinate, calculate the area under the ROC curve (AUC) of each experimental result. Generally, the larger the AUC, the higher the diagnostic value. The ROC curve analysis in this study showed that the corresponding AUCs of $\mathrm{MCH}, \mathrm{MCV}$, and $\mathrm{HGB}$ of $\mathrm{Li}$ and Han nationalities were all less than 0.5 , indicating that the values of $\mathrm{MCH}, \mathrm{MCV}, \mathrm{HGB}$ and other parameters in blood cell analysis have poor diagnostic value for thalassaemia screening. Related research reports such as Xie Yuping [12] and others in the Fujian area pointed out that $\mathrm{MCV}<81.25 \mathrm{fL}, \mathrm{MCH}<27.30 \mathrm{pg}, \mathrm{HGB}_{\text {male }}<128.5 \mathrm{~g} / \mathrm{L}$ and $\mathrm{HGB}_{\text {female }}<123.5 \mathrm{~g} / \mathrm{L}$ can be used as the best cut-off value for screening $\alpha$-thalassaemia. The calculated AUC are 0.99, 0.994 and 0.959, respectively, and have high sensitivity and specificity. After analyzing the mild $\alpha$-thalassaemia group, Feng Guiling et al. [13] found that the cut-off values of MCV and MCH were $74.9 \mathrm{fL}, 24.65 \mathrm{pg}$, and AUC were 0.962 and 0.961, respectively. Compared with the former, the results of this study are quite different, which may be related to the different choice of subjects and the more patients with anemia in the normal control group. However, clinical blood cell analysis is not easy to distinguish between thalassemia patients and other anemia patients.

In the related research on thalassaemia screening by hemoglobin electrophoresis, the domestic Zhang et al. [14] research results in Yunnan area showed that the optimal threshold for $\beta$-thalassemia screening is $\mathrm{HbA} 2 \geq 4.0 \%$, and $\mathrm{HbA} 2$ has a higher effect on $\beta$-thalassemia Diagnostic value. In this study, the ROC curves of $\mathrm{HbA}$ for $\alpha$-thalassaemia screening in $\mathrm{Li}$ and $\mathrm{Han}$ nationalities both showed: $0.5<$ AUC $<0.6$, indicating that although it has a certain value in the diagnosis of $\alpha$-thalassaemia, the effect is average. The ROC curve of HbA2 for $\beta$-thalassaemia screening showed that: AUC $>0.95$, indicating that it has a better diagnostic effect on $\beta$-thalassaemia. In summary, the best cut-off value in this study can be obtained: In the screening of $\alpha$-thalassaemia, the Li nationality has $\mathrm{HbA}>96.95 \%$ as the suspected $\alpha$-thalassaemia carrier, and the Han nationality 
has $\mathrm{HbA}>97.75 \%$ as the suspected $\alpha$-thalassaemia carrier. In $\beta$-thalassaemia screening, the Li nationality had $\mathrm{HbA} 2>4.20 \%$ as suspected $\beta$-thalassemia carriers, and the Han nationality had $\mathrm{HbA} 2>3.45 \%$ as suspected $\beta$-thalassemia carriers.

\section{Fund Program}

The Youth Science and Technology Talents Academic Innovation Program Project of Hainan Association for Science and Technology (QCXM201921)

\section{Conflicts of Interest}

The authors declare no conflicts of interest regarding the publication of this paper.

\section{References}

[1] Hu, J.J., Chen, X.P., Wang, J., et al. (2018) The Prevalence of Thalassemia and Rare Genotype Detection in Middle School Students of Li Nationality in Hainan. Guangdong Medicine, 39, 1471-1477.

[2] Devanesan, S., AlQahtani, F., AlSalhi, M.S., et al. (2019) Diagnosis of Thalassemia Using Fluorescence Spectroscopy, Auto-Analyzer, and Hemoglobin Electrophoresis: A Prospective Study. Journal of Infection and Public Health, 12, 585-590. https://doi.org/10.1016/j.jiph.2019.02.018

[3] Viprakasit, V. and Ekwattanakit, S. (2018) Clinical Classification, Screening and Diagnosis for Thalassemia. Hematology/Oncology Clinics of North America, 32, 193-211. https://doi.org/10.1016/j.hoc.2017.11.006

[4] Zhang, W.Y., Yang, X.P., Li, L.H., et al. (2016) Application of MCH, MCV, RDW-CV, HbA2 and Erythrocyte Fragility Test in the Differential Diagnosis of Thalassemia and Iron Deficiency Anemia. Biotechnology Communications, 27, 244-248.

[5] Tan, S., Li, Q.H., Chen, X., et al. (2018) The Clinical Value of Thalassemia Screening. Laboratory Medicine, 33, 730-733.

[6] Helmi, N., Bashir, M., Shireen, A., et al. (2017) Thalassemia Review: Features, Dental Considerations and Management. Electron Physician, 9, 4003-4008.

https://doi.org/10.19082/4003

[7] Urrechaga, E., Aguirre, U. and Izquierdo, S. (2013) Multivariable Discriminant Analysis for the Differential Diagnosis of Microcytic Anemia. Anemia, 2013, Article ID: 457834. https://doi.org/10.1155/2013/457834

[8] Uulen, H., Hanimeli, O., Karaca, O., et al. (2012) $\alpha$-Thalassemia Frequency and Mutations in Children with Hypochromic Microcytic Anemias and Relation with $\beta$-Thalassemia, Iron Deficiencyanemia. Pediatric Hematology and Oncology, 29, 241246. https://doi.org/10.3109/08880018.2012.661831

[9] Hu, J.J., Chen, X.P., Wang, J., et al. (2018) Application of Blood Cell Analysis and Hemoglobin Electrophoresis in the Screening of Thalassemia in Li Ethnic Group. Chongqing Medical Journal, 47, 3776-3779.

[10] Lin, J.D., Li, J.H., Zhu, Z.J., et al. (2014) ROC Curve to Evaluate the Value of Red Blood Cell Parameters in the Screening of Early Pregnant Women for Thalassemia. Guangdong Medicine Journal, 35, 1397-1399. 
[11] He, T.W., Yu, L.H., Guo, H., et al. (2016) ROC Curve Analysis of the Value of Hemoglobin A2 in Screening for Thalassemia. Chinese Journal of Experimental Hematology, 24, 1828-1832.

[12] Xie, Y.P., Cao, Y.P., Zhu, X.J., et al. (2019) The Value of Hematological Indicators in the Screening of Alpha-Thalassemia in Fujian, China. Chinese Journal of Experimental Hematology, 27, 165-169.

[13] Feng, G.L., Zhu, X.L., Wang, Z.Y., et al. (2015) The Clinical Application of Red Blood Cell Index and HbA2 in the Screening of Mild Alpha Thalassaemia. Journal of Tropical Medicine, 15, 1069-1072.

[14] Zhang, J., He, J., Mao, X., et al. (2017) Haematological and Electrophoretic Characterisation of $\beta$-Thalassaemia in Yunnan Province of Southwestern China. BMJ Open, 7, e013367. https://doi.org/10.1136/bmjopen-2016-013367 